\title{
Análise da Função Diastólica do Ventrículo Esquerdo em Hipertensos sob Variação de Condições Hemodinâmicas Provocadas: Estudo Comparativo entre o Doppler Transmitral e Tissular
}

\author{
Assessment of Left Ventricular Diastolic Function in Hypertensive Patients Under \\ Different Hemodynamic Conditions: Comparative Study Between Transmitral and Tissue \\ Doppler Echocardiography
}

Luiz Cláudio Danzmann, Valéria Centeno de Freitas, Luiz Felipe Araújo, Marco Antonio Rodrigues Torres Hospital de Clínicas de Porto Alegre e Universidade Federal do Rio Grande do Sul - Porto Alegre, RS

\section{Objetivo}

Avaliar o comportamento das velocidades de movimentação diastólica do segmento VE septal posterior basal justa-anular, obtidos pelo Doppler tissular em relação às velocidades do fluxo transmitral de hipertensos, submetidos à sobrecarga de pré-carga por elevação dos membros inferiores por 5 minutos, e durante 1 minuto de handgrip (condições 1 e 2, respectivamente).

\section{Métodos}

Vinte e cinco pacientes ambulatoriais (15 homens, $52 \pm 11$ anos), com hipertensão arterial sistêmica >3 anos, com as seguintes variáveis do Doppler transmitral: $E, A$ e E/A, e do Doppler tissular: E', A' e E'/A', obtidas na situação basal e nas condições 1 e 2 . A comparação dos resultados (basal vs. condições 1 e 2) foi realizada por teste t pareado.

\section{Resultados}

$\mathrm{E}(\mathrm{cm} / \mathrm{s})$ variou de $68,88 \pm 11,94$ para $75,82 \pm$ 15,71 * enquanto $E^{\prime}(\mathrm{cm} / \mathrm{s})$ variou de $8,22 \pm 2,30$ para $8,31 \pm 2,24$ na condição $1(p<0,05)$. Adicionalmente, a comparação dos percentuais (\%) de variação dos índices de Doppler mitral com os \% de seus correspondentes medidos por Doppler tissular revelou diferença significativa entre $E$ e E', com incremento da pré-carga $(p=0,01)$.

\section{Conclusão}

Em pacientes com VE adaptado à hipertensão arterial sistêmica, o índice E' modificou-se menos do que o índice E após manobra de elevação de pré-carga, o que deve orientar no sentido de sua maior utilização na avaliação da função diastólica por ecocardiografia.

\section{Palavras-chave}

Função diastólica ventricular esquerda, hipertensão arterial, Doppler tissular.

\section{OBJECTIVE}

To evaluate the behavior of $L V$ diastolic displacement velocities of basal posterior septum near mitral ring segment obtained by tissue Doppler in relation to mitral flow velocities of hypertensive subjects, submitted to preload increase by left inferior limbs elevation for 5 minutes, and during 1 minute of handgrip (conditions 1 and 2, respectively).

\section{Methods}

Twenty five outpatients (15 men, $52 \pm 11$ years), with arterial hypertension $>3$ years, by the transmitral flow Doppler variables: $E, A$ and $E / A$, and of tissue Doppler: $E^{\prime}, A^{\prime}$ and $E^{\prime} / A^{\prime}$, in basal situation and in conditions 1 and 2. For comparison of results in basal situation with conditions 1 and 2 a paired test was applied.

\section{RESULTS}

$E(\mathrm{~cm} / \mathrm{s})$ varied from $68,88 \pm 11,94$ to $75,82 \pm$ 15,71 * and $E^{\prime}(\mathrm{cm} / \mathrm{s})$ varied from $8,22 \pm 2,30$ to $8,31 \pm$ 2,24 in condition $1(p<0.05)$. In addition, a comparison of variation percent (\%) of Doppler transmitral indices with corresponding tissue Doppler indices variation \% showed a significant difference between $E$ and $E^{\prime}$, with preload increase $(p=0.01)$.

\section{Conclusion}

Patients with left ventricular adaptation to systemic arterial hypertension showed less modification of the index $E^{\prime}$ than $E$, after preload increase maneuver, an evidence that may suggest its more ample utilization in the context of echocardiographic left ventricular diastolic function evaluation.

\section{KEY WORDS}

Left ventricular diastolic function; arterial hypertension; tissue Doppler. 
A disfunção diastólica ventricular esquerda (VE) tem sido reconhecida como uma das principais causas primárias de insuficiência cardíaca congestiva (ICC) ${ }^{1}$. Diversos estudos indicam que 30 a $50 \%$ dos pacientes com a síndrome de ICC mantêm a função sistólica ventricular esquerda normal ou pouco comprometida, gerando, como propõem alguns, a denominação de insuficiência cardíaca "diastólica" para este grupo de pacientes ${ }^{2}$. Desde então, o perfil dos fluxos através da valva mitral na via de entrada do VE tem sido avaliado rotineiramente, através da análise do Doppler espectral, na busca de padrões que caracterizem tal diagnóstico e informem algo sobre o prognóstico desta doença ${ }^{3}$.

Embora tal método tenha estabelecido inicialmente o que seria chamado padrão de medida diastólica, logo se verificou que os resultados das avaliações eram variáveis e nem sempre reproduzíveis ${ }^{4}$. Alguns estudos mostraram, inclusive, ausência de um padrão de normalidade diante de um quadro clínico evidente de disfunção diastólica e insuficiência cardíaca, o que passou a ser denominado "padrão pseudonormal" ${ }^{5}$. Tal padrão resulta do incremento da pressão atrial esquerda, que se torna superior aos efeitos do comprometimento do relaxamento do ventrículo esquerdo. Esta dependência da velocidade e do tempo de relaxamento isovolumétrico em relação ao relaxamento do VE, bem como à pressão atrial esquerda, foi evidenciada há muito tempo, e estas variáveis estabeleceram uma moldura que expressa as limitações dos métodos que se baseiam na análise do fluxo transmitral ${ }^{5}$. Este padrão pode ocorrer, porque as variáveis do fluxo da via de entrada do VE são dados de velocidades determinados pela diferença de pressão entre o átrio esquerdo e o VE na diástole. A avaliação das mudanças de volume tem a vantagem teórica de ser menos dependente da pré-carga do que as variáveis do DTM6.

O Doppler tissular (DT) estuda os sinais de alta amplitude e baixa velocidade derivados do miocárdio ${ }^{7}$. Este recurso tem se mostrado útil como um índice quantitativo da função segmentar do VE, tanto para a análise da diástole $^{8}$ quanto da sístole ${ }^{9}$. Em estudos de disfunção diastólica, observou-se que este apresenta relativa independência da pré-carga ${ }^{6}$, tendo sido documentado haver pouca probabilidade de ele mostrar o padrão de 'pseudonormalização' visto com emprego do DTM.

A avaliação da função diastólica pelo DT pode, inclusive, ser utilizada para a análise diastólica segmentar com um alto nível de acurácia, conforme demonstrado em estudo recente ${ }^{10}$, no qual a interrogação dos múltiplos locais através desta técnica pôde ser feita usando-se os dados do Doppler tissular em cores, processando-se os sinais de todos os segmentos de VE.

O presente estudo clínico foi projetado com o fim de avaliar o comportamento dos índices diastólicos de VE extraídos de DT em relação às de DTM sob a influência da sobrecarga hemodinâmica - induzida pela modificação da pré-carga (relacionada à elevação dos membros inferiores) - e da pós-carga - pelo exercício isométrico com a manobra de handgrip - durante a análise da função diastólica desta câmara. A população estudada compunha-se de portadores de hipertensão arterial sistêmica (HAS) há pelo menos três anos, cujos ventrículos esquerdos encontravam-se adaptados a esta condição.

\section{Métodos}

Seleção dos pacientes - Foram estudados consecutivamente vinte e cinco pacientes ambulatoriais, com HAS há pelo menos três anos. Os critérios de exclusão foram: sinais clínicos, eletrocardiográficos ou ecocardiográficos de isquemia miocárdica; doenças valvares; arritmias cardíacas; cardiomiopatias e doenças pericárdicas. Além disso, pacientes renais crônicos e diabéticos também foram excluídos. Não houve interferência dos pesquisadores com o uso das medicações cardioativas para tratamento da HAS. Todos os indivíduos que fizeram o teste para fins do presente estudo deram consentimento, por escrito, pós-informação, para entrar no protocolo, o qual foi aprovado pelo Comitê de Ética Institucional com o título de "Análise da função diastólica do ventrículo esquerdo por imagem de Doppler pulsátil tissular em pacientes hipertensos" na reunião do dia 19.01.2001 sob o número 99376.

Ecocardiografia - Os ecocardiogramas foram realizados em repouso, na posição de decúbito lateral esquerdo, usando-se um equipamento disponível comercialmente (HDI 5000, Philips-ATL, Seattle, WA). As imagens foram obtidas nos cortes tomográficos estandardizados do VE (parasternal longitudinal e transversais, e apicais 4-, 5-, e 2- câmaras) usando um transdutor de 3,5 MHz no modo harmônico. As imagens foram arquivadas em fitas VHS, e todas as análises do Doppler espectral mitral e tissular, bem como as medidas das cavidades e análises da cinética do VE foram realizadas on-line e off-line. As espessuras parietais de septo interventricular (Psiv) e parede posterior (Pp), bem como os diâmetros diastólico (Dd) e sistólico (Ds) do VE foram obtidos usando-se o modo-M; a hipertrofia VE (HVE) foi definida como um índice de massa $\geq 110 \mathrm{~g} / \mathrm{m}^{2}$ para mulheres e $125 \mathrm{~g} / \mathrm{m}^{2}$ para homens; e a dimensão $\mathrm{AE}$ foi medida durante a sístole tardia. A massa ventricular foi calculada automaticamente pelo equipamento (Fórmula da massa $\left.\mathrm{VE}=1,04 \times\left[(\mathrm{Dd}+\mathrm{Pp}+\mathrm{Psiv})^{3}-\mathrm{Dd}^{3}\right]\right)$ e os volumes sistólico e diastólico do VE, bem como a fração de ejeção, foram obtidos usando-se o método de Teichholz.

Velocidades de fluxo transmitral - As imagens transtorácicas apicais 4-câmaras do VE foram registradas usando-se o equipamento padronizado com um transdutor de $2.5 \mathrm{MHz}$. A amostra de volume foi colocada nos bordos livres dos folhetos da válvula mitral, na vista apical 4-câmaras. A velocidade do fluxo foi registrada com o método do Doppler pulsátil, usando-se a média de três batimentos representativos. O pico da velocidade diastólica precoce $(E)$, o pico da velocidade sistólica atrial (A) e a razão $E / A$ foram determinados. 
Doppler tissular - As velocidades miocárdicas foram medidas pela ativação da função do Doppler tissular em cores, a fim de se registrarem as baixas velocidades e os sinais de alta intensidade, fazendo-se uso de alto número de frames necessário (120 MHz), com uma resolução temporal de $8 \mathrm{~ms}$. Após tal período, posicionouse a amostra volume na porção basal septal posterior e registrou-se o padrão do pico da velocidade precoce (E' cm/ s), o pico da velocidade correspondente à sístole atrial, ou enchimento diastólico tardio ( $\left.A^{\prime} \mathrm{cm} / \mathrm{s}\right)$, e a razão E'/A'11.

Modificações induzidas nas condições hemodinâmicas - Os perfis das velocidades foram obtidos em condição basal, após manobra de aumento da pré-carga ao VE (condição 1) - a qual consistiu em elevação uniforme e sustentada de ambos os membros inferiores a um angulo de $45^{\circ}$ em relação ao leito, durante um período de cinco minutos contínuos -, e após manobra de aumento de póscarga ao VE (condição 2) - a qual consistiu em um minuto contínuo a 30\% da capacidade máxima de apreensão de dinamômetro manual - handgrip.

Reprodutibilidade - A variabilidade interobservador - calculada como a diferença de duas medidas do mesmo paciente por dois observadores diferentes e igualmente treinados, divididas pelo valor médio desta - variou de 1,5 a 2,6\% para os índices do fluxo transmitral e de 0,8 a 1,7\% para o Doppler pulsátil tissular.

Análise estatística - Todos os valores foram apresentados como média \pm desvio padrão (DP). As variáveis obtidas pelo Doppler mitral e pelo Doppler tissular em repouso (basal) foram comparadas com a condição associada à elevação dos membros inferiores (condição 1) e com a associada à manobra de handgrip (condição 2). As diferenças das médias de DTM E DT entre situação basal e condições 1 e 2 foram comparadas por teste t pareado de Student. Em uma segunda análise, foi calculado o percentual de variação dos índices de DTM nas condições 1 e 2, em relação ao basal, e comparado aos respectivos valores percentuais (\%) de variação dos índices de DPT nas condições 1 e 2, também em relação ao basal. Neste momento constatou-se que os valores médios da diferença de percentual de variação $(\Delta \%)$ eram menores do que 0 desvio padrão. Ao se concluir que os dados não tinham distribuição normal, utilizou-se o teste t de Wilcoxon para a comparação destas variáveis. Um valor de $P<0,05$ foi considerado estatisticamente significativo.

\section{Resultados}

Características da amostra - A amostra foi composta por vinte e cinco pacientes com idades entre 32 e 75 anos (52 $\pm 10,9$ ), com índices antropométricos limítrofes (Índice de massa corporal=27,9) e não apresentaram variações extremas de pressão arterial media ou freqüência cardíaca durante o exame ( $P A M=108 \pm 20,7 \mathrm{mmHg}$ e $F C=69,7 \pm 13,1 \mathrm{bpm}$ ). Os índices médios de função sistólica foram normais, com fração de ejeção de $66,9 \pm 9,6 \%$ e fração de encurtamento sistólico de $37,5 \pm 7,1 \%$ e massa ventricular esquerda

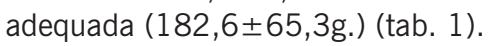

\begin{tabular}{|lc|}
\hline \multicolumn{3}{|c|}{ Tabela 1 - Dados clínicos, ecocardiográficos e } \\
antropométricos da amostra
\end{tabular}

Valores basais - Os valores, em condições basais, para as velocidades calculadas pelo DTM foram: $E=68,88 \pm$ $1,94 \mathrm{~cm} / \mathrm{s}, A=61,88 \pm 12,99 \mathrm{~cm} / \mathrm{s}, E / A=1,16 \pm 0,31$. Os valores, em condições basais, para as velocidades calculadas pelo DT foram: $E^{\prime}=8,31 \pm 2,31 \mathrm{~cm} / \mathrm{s}, A^{\prime}=$ $9,12 \pm 1,93 \mathrm{~cm} / \mathrm{s}, E^{\prime} / A^{\prime}=0,92 \pm 028$ (Tabela 2).

Dependência das velocidades do fluxo mitral em relação à mudança das condições hemodinâmicas - O perfil de velocidade do índice DTM-E variou de maneira significativa, mediante a elevação da pré-carga $(68,88 \pm 11,9 \mathrm{~cm} / \mathrm{s}$ vs. $75,82 \pm 15,71 \mathrm{~cm} / \mathrm{s}, \mathrm{P}=0,002)$ (fig. 1). Não houve variação significativa de nenhum dos itens do DTM com elevação da pós-carga. 0 índice DTE' não apresentou variação significativa após a elevação da pré-carga $(8,22 \pm 2,30 \mathrm{~cm} / \mathrm{s}$ vs. $8,31 \pm 2,24 \mathrm{~cm} / \mathrm{s}$, $\mathrm{P}=\mathrm{NS}$ ) (fig. 2). Em contraposição, o índice $A^{\prime}$ e a razão E'/A' apresentaram alteração significativa $(9,12 \pm$ $1,93 \mathrm{~cm} / \mathrm{s}$ vs. $10,05 \pm 2,36 \mathrm{~cm} / \mathrm{s}, \mathrm{P}=0,01)$ e $(0,92 \pm 0,28$ vs. $0,86 \pm 0,25, P=0,03)$, respectivamente. Com 0 incremento da pós-carga, E' e a relação E'/A' alteraramse $(8,22 \pm 2,30 \mathrm{~cm} / \mathrm{s}$ vs. $7,54 \pm 1,86 \mathrm{~cm} / \mathrm{s}, P=0,04)$ e $(0,92 \pm 1,93 \times 0,81 \pm 1,27, \mathrm{P}=0,01)$.

Comparação entre variações do Doppler mitral e variações do Doppler tissular - A comparação entre os percentuais de variação das velocidades do fluxo mitral diante da mudança das condições hemodinâmicas com os mesmos percentuais de variação obtidas pela análise do Doppler tissular revelou uma diferença significativa de variação entre os $\Delta \%$ para a onda $E$ vs. $\Delta \%$ para a onda $\mathrm{E}^{\prime}(6,1 \%$ vs. $0 \%, \mathrm{P}=0,01)$, confirmando 0 comportamento de invariabilidade de E' para incremento de pré-carga (Tabela 3).

Comparando-se a magnitude das mudanças observadas nas variáveis estudadas pelos dois métodos, sob as condições hemodinâmicas basais, elevação dos membros inferiores e handgrip, e aplicando-se o teste t de Wilcoxon para se avaliarem as diferenças entre as medianas das aferições, fica evidente haver uma diferença significativa entre as variações das ondas $\mathrm{E}$ do Doppler mitral em relação às ondas E' do Doppler tissular. 


\begin{tabular}{|c|c|c|c|c|c|c|}
\hline & \multicolumn{3}{|c|}{ Doppler de fluxo mitral } & \multicolumn{3}{|c|}{ Doppler tissular } \\
\hline & E & A & $E / A$ & $E^{\prime}$ & $A^{\prime}$ & $E^{\prime} / A^{\prime}$ \\
\hline & Média \pm DP & Média \pm DP & Média \pm DP & Média \pm DP & Média \pm DP & Média \pm DP \\
\hline Basal & $68,88 \pm 11,94$ & $61,88 \pm 12,99$ & $1,16 \pm 0,31$ & $8,22 \pm 2,30$ & $9,12 \pm 1,93$ & $0,92 \pm 0,28$ \\
\hline Condição1 & $75,82 \pm 15,71^{*}$ & $63,34 \pm 16,61$ & $1,30 \pm 0,52$ & $8,31 \pm 2,24$ & $10,05 \pm 2,36^{*}$ & $0,86 \pm 0,25^{*}$ \\
\hline Condição2 & $70,30 \pm 17,49$ & $66,44 \pm 17,55$ & $1,14 \pm 0,47$ & $7,54 \pm 1,86^{*}$ & $9,60 \pm 2,20$ & $0,81 \pm 0,27^{*}$ \\
\hline
\end{tabular}

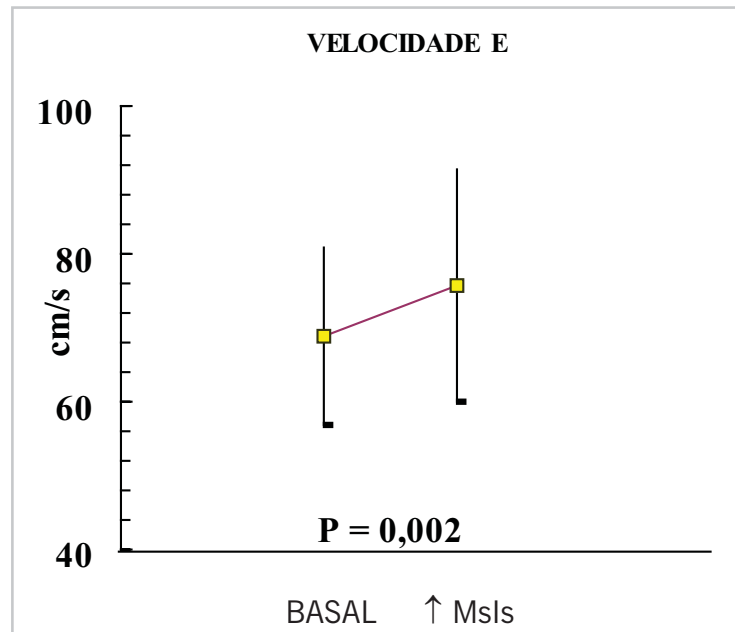

Fig. 1 - Modificação na velocidade de fluxo transmitral E em situação basal e após elevação da pré-carga ao VE por inclinação dos membros inferiores a $45^{\circ}$.

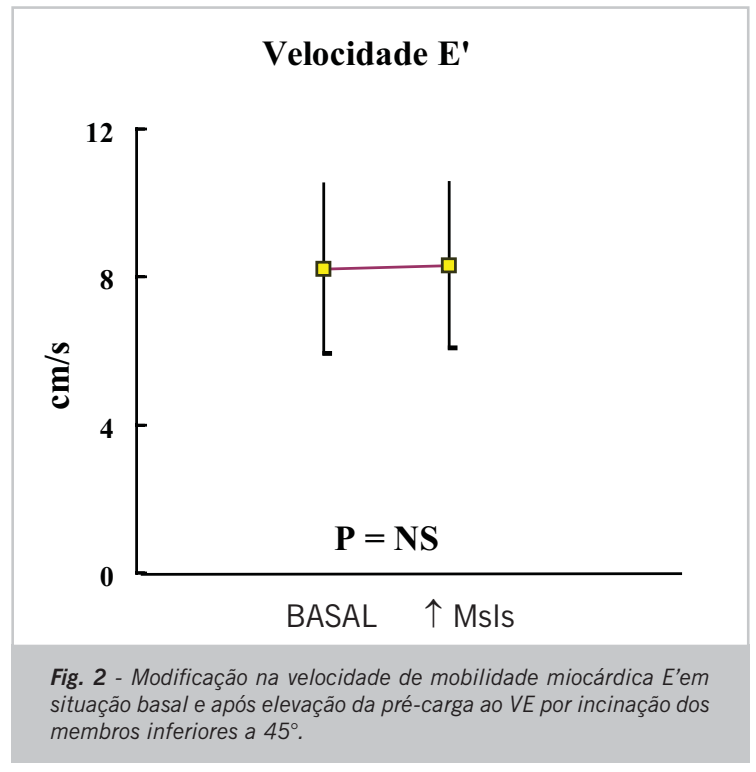

Tabela 3 - Diferenças entre percentuais de variação $(\Delta \%)$ dos índices de DTM e DPT nas condições de elevação de pré e pós-carga (C.1 e C.2, respectivamente) em relação ao basal

$\begin{array}{ccc}\text { DTM } & \text { DT } & \mathbf{P} \\ 6,1 & 0,0 & 0,010 \\ 1,1 & -7,1 & 0,045 \\ -1,2 & 10,0 & \text { NS } \\ 10,7 & 8,2 & \text { NS } \\ 8,0 & -6,3 & 0,001 \\ -8,0 & -14,3 & \text { NS }\end{array}$

$\begin{array}{lc}\Delta \% \text { E-E' C.1/basal } & 6,1 \\ \Delta \% \text { E-E' C.2/basal } & 1,1 \\ \Delta \% \text { A-A' C.1/basal } & -1,2 \\ \Delta \% \text { A-A' C.2/basal } & 10,7 \\ \Delta \% \text { E/A-E' } / A^{\prime} \text { C. } 1 / \text { basal } & 8,0 \\ \Delta \% \text { E } / \text { A-E' } / A^{\prime} \text { C.2/basal } & -8,\end{array}$

$\Delta \%$ E-E' C.1/basal - diferença de percentuais de variação das ondas $E$ e E'após elevação de pré-carga; $\Delta \%$ E-E' C.2/basal - diferença de percentuais de variação das ondas $E$ e E'após elevação de pós-carga; $\Delta \% A$ - $A^{\prime} C .1 /$ basal - diferença de percentuais de variação das ondas $A$ e $A^{\prime}$ após elevação de pré-carga; $\triangle \% A$-A' C.2/basal - diferença de percentuais de variação das ondas $A$ e A'após elevação de pós-carga; $\triangle \%$ E/A-E'/A' C. $1 /$ basal - diferenças de percentuais de variação das razões $E / A$ e E'/A' após a elevação da pré-carga; $\Delta \% E / A-E^{\prime} / A^{\prime} C .2 /$ basal - diferenças de percentuais de variação das razões $E / A$ e E'/A' após a elevação da pós-carga.

\section{Discussão}

Diástole e interferência da pré-carga e da pós-carga - O método mais tradicional para aferir a diástole é a ecocardiografia com uma análise do fluxo transmitral por Doppler pulsátil. Tal teste, entretanto, apresenta uma variação importante dos índices das ondas $\mathrm{E}$ e $\mathrm{A}$ e da relação de ambas as ondas (E/A), diante de modificações da pré-carga ao VE.

O Doppler tissular é o método que avalia a velocidade miocárdica segmentar na diástole e também na sístole. Em um estudo utilizando DT do miocárdio septal interventricular em relação ao fluxo transmitral em vinte pacientes com padrão de déficit de relaxamento (relação ondas $E / A<1$ ), submetidos à infusão de $700 \mathrm{ml}$ de soro fisiológico, evidenciou-se significativa modificação da relação das velocidades E/A medida por fluxo transmitral $(E / A=0,7 \pm 0,1$ vs. $0,9 \pm 0,1 p<0,01)$, o que não foi observado nos parâmetros medidos por DPT ( $E^{\prime} / A^{\prime}=0,5$ $\pm 0,1$ vs. $0,5 \pm 0,1, p=N S)^{6}$.

Estes resultados apontam para o fato de que o exame da mobilidade de segmentos da parede ventricular por DPT é relativamente independente da pré-carga ventricular, conforme já havia sido previamente sugerido ${ }^{12}$. 
Em relação à pós-carga, tanto o método de estudo do fluxo transmitral quanto o DPT exibem variações. Oki e cols. ${ }^{13}$, estudaram a fundo o Doppler pulsátil tissular. Em uma publicação ${ }^{13}$, os referidos autores avaliaram, em vinte voluntários sadios, a função sistólica e diastólica, através do ecocardiograma, antes e depois de elevação aguda da póscarga secundária à infusão de angiotensina II (até obterem elevação de 30 mmHg na pressão arterial média). Agindo de tal maneira, observaram que todos os parâmetros diastólicos (DTM e DPT) sofreram variações significativas.

Estes achados sugeriram que ambos os métodos não são absolutamente independentes da pós-carga. Este fato, todavia, possibilita sua utilização para detectar e acompanhar o efeito de variações da pós-carga no VE, o que poderia ser útil, por exemplo, para acompanhamento da evolução clínica ou, eventualmente, para a quantificação da resposta às terapias medicamentosas.

Movimentação do anel mitral e dos segmentos adjacentes do miocárdio ventricular - Diante da manutenção de uma posição relativamente fixa do ápice ventricular durante o ciclo cardíaco ${ }^{14}$ - devendo - VE estar relativamente preservado quanto à sua geometria -, as modificações no eixo longitudinal refletiriam as modificações volumétricas, como apontado por Sohn e cols. ${ }^{6}$. No presente estudo, foi escolhida propositadamente a análise da movimentação do segmento septal interventricular na sua porção basal, tendo em vista que esta porção miocárdica adjacente ao anel mitral move-se mais paralelamente que os outros (como a face anterior, lateral e inferior), conforme já destacado por Sohn e cols. ${ }^{6}$.

Conforme se observa pela Figura 3, o Doppler tissular captou, no segmento analisado, dois movimentos distintos em direção à câmara atrial: um denominado ( $\left.E^{\prime}\right)$, o qual inicia simultaneamente com o começo do fluxo para a via de entrada do VE, onde sua velocidade de pico é sempre mais precoce que o pico da velocidade do fluxo pelo Doppler mitral e termina bem antes do final do influxo de sangue pelo orifício mitral. O pico da velocidade E' é mais elevado que o pico de uma outra onda ( $\left.A^{\prime}\right)$, o que se assemelha ao padrão normal do fluxo transmitral. Como salientado por Sohn e cols. ${ }^{6}$, em seu clássico estudo, Mitral inflow after the end of left ventricular lengthening in the long-axis dimension, whether it is driven by pressure gradient or inertia, would lead to an increase in left ventricular volume in the short-axis dimension, which would mean higher compliance of the ventricular chamber in the short-axis dimension. Therefore, it may be assumed that a relaxation abnormality reflected in the long-axis dimension could potentially be evident earlier than clinical manifestation of global left ventricular relaxation abnormality. Como uma conseqüência deste particular de tal metodologia, assume-se que, ao se analisarem as variáveis pelo Doppler mitral, estar-se-á diante de dados relativos às velocidades, ao passo que, no caso do Doppler tissular, estes dados serão relativos às alterações de volume.

Doppler tissular: independência das modificações da pré-carga - $\mathrm{Na}$ análise dos dados do presente estudo encontramos independência dos valores de E' às manobras de elevação dos membros inferiores (condição 1 , correspondendo à variação da pré-carga). Previamente, outros investigadores - como Garcia e cols. ${ }^{12}$ - já haviam observado que a velocidade $E^{\prime}$ pouco se correlacionava com a velocidade do pico $E$, fato que apontava para uma relativa independência do pico da velocidade $E^{\prime}$ obtida pelo Doppler tissular. Com o presente estudo, demonstra-se originalmente que, mesmo com manobras mais simples e não-invasivas, conduzidas à beira do leito, não se observam modificações significativas no pico precoce da velocidade $E^{\prime}$ do anel mitral ao Doppler tissular (em contraposição à velocidade $\mathrm{E}$ do Doppler mitral), após uma alteração da pré-carga como a elevação dos membros inferiores.

Manobras de elevação dos membros inferiores e de handgrip para estudar as adaptações do coração às novas condições hemodinâmicas - Ambas as manobras utilizadas no presente estudo, para fins de provocar novos patamares hemodinâmicos como uma maneira de testar as respostas adaptativas do coração e da circulação, vêm sendo empregadas há muito tempo. 0 mecanismo de aumento da pré-carga com a conseqüente elevação da pressão arterial sistêmica foi recentemente

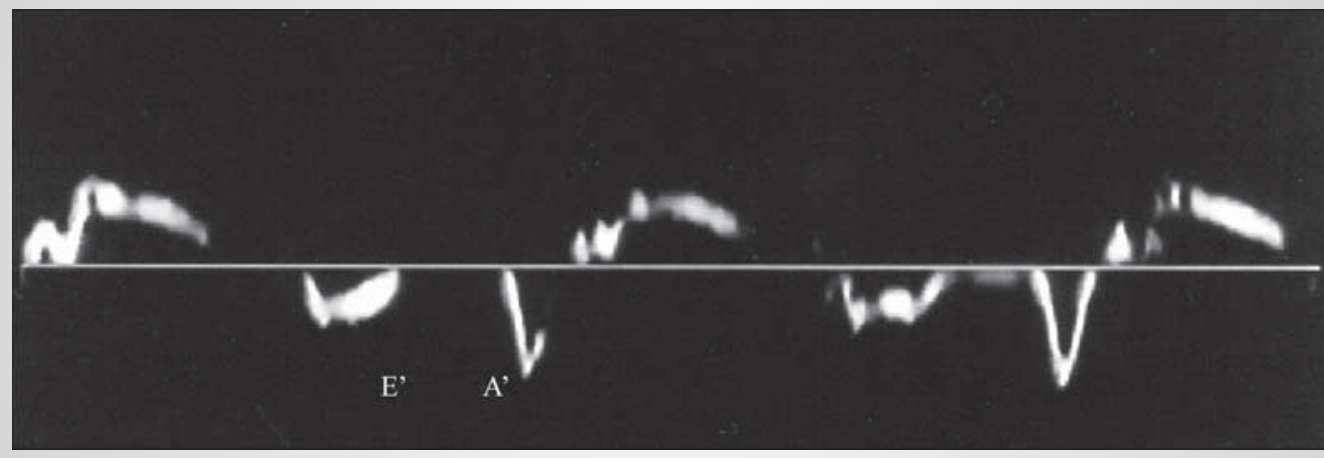

Fig. 3 - Registro das ondas de mobilidade diastólica miocárdicas E' e A' por Doppler pulsátil tissular. 
relacionado à liberação de potentes vasoconstritores com ação endotelial ${ }^{15}$. O mecanismo de modificação hemodinâmica com aumento da pré-carga pela elevação dos membros inferiores, utilizando-se o protocolo que aplicamos aos indivíduos do presente estudo, também já está estabelecido ${ }^{16}$. Os comentários em relação à magnitude que tais manobras podem induzir nestas variáveis hemodinâmicas merecem ser analisados. Conforme se observa nas figuras 1 e 2 (com > pré-carga), as alterações foram de suficiente magnitude para interferir no comportamento da variável $E$ analisada, obtidas pelo Doppler mitral no presente estudo, porém não alteraram a variável E' medida pelo Doppler tissular. Tak e cols. observou efeito significativo da manobra de handgrip sobre a pré-carga e, conseqüentemente sobre índices diastólicos ${ }^{17}$. Outras publicações ratificaram o uso da manobra para produção de exercício isométrico e elevação efetiva de pós-carga, utilizando uma força aplicada de 30 a $50 \%$ da força máxima num período de 1 a $5 \min ^{18,19}$. Observamos que alguns pacientes tiveram dificuldades na manutenção da mesma força do handgrip pelo tempo que estipulamos (cinco minutos), e alguns indivíduos suportaram apenas uma carga menor e por menos tempo, muito embora este seja um problema comum em estudos previamente publicados com a utilização de similar tipo de exercício isométrico.

Diagnóstico da disfunção diastólica pelo Doppler tissular do segmento basal do septo interventricular - Os achados do presente estudo apontam para uma independência da pré-carga, considerando-se a velocidade da onda E'. Os dados e os resultados estatísticos que foram derivados pela análise de todas as variáveis estão de acordo com os achados presentes na literatura ${ }^{6,12,13,16}$.

Aplicações na prática clínica dos achados do presente estudo - Nos pacientes com o padrão de 'pseudonormalização', cujos parâmetros são obtidos tanto pelo Doppler mitral quanto pelo tissular, a variação dos picos das velocidades $E$ e E', bem como a razão E/A em relação à razão $E^{\prime} / A^{\prime}$, pode ser empregada para se diferenciar este padrão 'pseudonormal' de um padrão de enchimento normal. Considerando-se que a maneira mais difundida na prática ecocardiográfica tem sido tentar diferenciar este padrão 'pseudonormal' do padrão da função diastólica normal através da análise do padrão dos fluxos nas veias pulmonares - e nem sempre ele é útil para este fim neste subgrupo de pacientes - , a adição das manobras simples que propomos nesta investigação pode ser proposta para o estudo desses pacientes. Além disso, a variação dos índices de DT após modificações nas condições de pós-carga verificada neste estudo, sugere que estes índices possam ser utilizados para monitorização da função diastólica de pacientes hipertensos em tratamento com medicações que reduzem a pós-carga.

Limitações do estudo - O presente estudo, a exemplo de outros previamente publicados, apresenta várias limitações práticas, dentre as quais se destacam as seguintes: 1) considerando-se que foram excluídos pacientes com dissinergias do ventrículo esquerdo, é possível que os pacientes com alterações da cinética (por exemplo, comprometendo o segmento basal do septo interventricular) venham a exibir uma anormalidade nos perfis das variáveis analisadas, o que não exprime o comportamento global do ventrículo, o qual pode ser normal para o comportamento do parâmetro da sua função diastólica ${ }^{16}$; 2) embora tenhamos optado por medir a velocidade miocárdica na região mais adjacente ao anel, no septo interventricular, correspondendo mais precisamente ao segmento basal do septo interventricular posterior, por estar submetido em menor grau ao movimento translacional do coração, esta abordagem tem a desvantagem de ser afetada sempre que a função ventricular direita é anormal, por exemplo, secundariamente à hipertensão pulmonar, principalmente se esta câmara exibir sinais de disfunção associada ${ }^{16}$; 3) como foi critério de exclusão que os pacientes não exibissem o ritmo sinusal, é possível que, nos portadores de fibrilação atrial, por exemplo, os presentes achados não sejam aplicáveis; 4) embora seja menos afetado do que a análise pelo Doppler mitral, o aspecto limitante de uma janela acústica inadequada tem de ser levado em consideração. A avaliação diastólica por Doppler em geral é afetada também por taquicardia, valvulopatias e movimento rotacional cardíaco ${ }^{20}$.

Conclusões - Em pacientes com o ventrículo esquerdo adaptado à hipertensão arterial, a movimentação diastólica do segmento miocárdico adjacente ao anel mitral medida por Doppler tissular foi menos dependente da pré-carga do que quando estudada pela técnica de Doppler do fluxo transmitral. Este fato sugere a validade do método como índice coadjuvante para avaliação da função diastólica de VE neste grupo de pacientes. Concluise também, que os índices de DT sofreram variações significativas mediante a variação da pós-carga e que poderiam ser utilizados para monitorização e seguimento da diástole após tratamento anti-hipertensivos com drogas redutoras da pós-carga.

\section{Potencial Conflito de Interesses}

Declaro não haver conflitos de interesses pertinentes.

\section{REFERÊNCIAS}

1. Vasan R S, Benjamin E J. Diastolic heart failure - no time to relax. Editorials. N Engl J Med. 2001; 344:56-59.

2. Grossman W. Diastolic dysfunction and congestive heart failure. Circulation. 1990;81 (Suppl III):III-1-7.

3. Appleton CP, Firstenberg MS, Garcia MJ, Thomas JD. The echoDoppler evaluation of left ventricular diastolic function. A current

perspective. Cardiol Clin. 2000;18:513-46.

4. Nishimura RA, Abel MD, Hatle LK, Tajik AJ. Assessment of the diastolic function of the heart: background and current application of Doppler echocardiography. Part II. Clinical studies. Mayo Clin Proc. 1989;64:977-90.

5. Nagueh SF, Middleton KJ, Kopelen HA, et al. Doppler tissue imaging: 
a noninvasive technique for evaluating of left ventricular relaxation and estimation of filling pressures. J Am Coll Cardiol. 1997;30:1527-33.

6. Sohn D, Chai I, Lee D, et al. Assessment of mitral annulus velocity by Doppler tissue imaging in the evaluation of left ventricular diastolic function. J Am Coll Cardiol. 1997;30:474-80.

7. Hatle L, Sutherland GR. Regional myocardial function--a new approach. Eur Heart J. 2000;21:1337-57.

8. Wilkenshoff U, Sovany A, Wigström L, et al. Regional mean systolic myocardial velocity estimation by real time color Doppler myocardial imaging: a new technique for quantifying regional systolic function. J Am Soc of Echocardiogr. 1998;11:683-692.

9. Pasquet A, Armstrong G, Beachler L, Lauer M, Marwick T. Use of segmental tissue Doppler velocity to quantitate exercise echocardiography. J Am Soc Echocardiog. 1999;12:901-12.

10. von Bibra H, Tuchnitz A, Klein A, et al. Regional diastolic function by pulsed Doppler myocardial mapping for the detection of left ventricular ischemia during pharmacologic stress testing. J Am Coll Cardiol. 2000;36:444-452

11. Pai R, Gill K. Amplitudes, durations, and timings of apically directed left ventricular myocardial velocities: their normal pattern and coupling to ventricular filling and ejection. J Am Soc Echocardiogr. 1998;11:105-11.

12. Garcia MJ, Thomas JD, Klein AL. New echocardiographic applications for the study of diastolic function. J Am Coll Cardiol. 1998;32:865-75.

13. Oki T, Fukuda K, Tabata T, et al. Effect of an acute increase in afterload on left ventricular regional wall motion velocity in healthy subjects. J Am Soc Echocardiogr. 1999;12:476-83.

14. Assmann PE, Slager CJ, Dreysse SG, van der Borden SG, Oomen JA, Roelandt JR. Two-dimensional echocardiographic analysis of the dynamic geometry of the left ventricle: the basis for an improved model of wall motion. J Am Soc Echocardiogr. 1988;1:393.405.

15. Mangieri E, Tanzilli G, Barilla F, Ciavolella M, Puddu PE, De Angelis $C$, et al. Handgrip increases endothelin-1 secretion in normotensive young male offspring of hypertensive parents. J Am Coll Cardiol. 1998;31:1362-6.

16. Shimizu Y, Masaaki U, Shimizu H, et al. Peak negative myocardial velocity gradient in early diastole as a noninvasive indicator of left ventricular diastolic function. J Am Coll Cardiol. 1998;32:1418-25.

17. Tak T, Choudhary RS, Chatterjee S, et al. Effect of loading conditions on Doppler-derived transmitral flow indices in normal subjects and patients with coronary artery disease. Echocardiography. 1992;9:467-474.

18. Campaniello M, Antonelli G, Di Venere N, et al. The echocardiographic assessment of the functional variations in the left ventricle induced by isometric stress in subjects with primary cardiomyopathy in the pre-dilated phase. Cardiologia. 1994;39:543-9.

19. Heng MK, Bai JX, Marin J. Changes in left ventricular wall stress during isometric and isotonic exercise in men. Am J Cardiol. 1998;62(10 pt 1):794-8.

20. Oki T, Tabata T, Yamada H, et al. Left ventricular diastolic properties of hypertensive patients measured by pulsed tissue Doppler imaging. J Am Soc Echocardiogr. 1998;11:1106-12. 